\title{
Bisphosphonate associated osteomyelitis of the jaw in patients with bony exposure: prevention, a new way of thinking
}

\author{
Arezo TARDAST ${ }^{1}$, Reine SJÖMAN² ${ }^{2}$ Sigbjørn LøES ${ }^{1}$, Jahan ABTAHI ${ }^{2}$
}

1- Department of Oral \& Maxillofacial Surgery, Bergen, Norway.

2- Department of Oral \& Maxillofacial Surgery, Linköping, University Hospital, Linköping, Sweden.

Corresponding address: Jahan Abtahi - Department of Oral \& Maxillofacial Surgery, Linköping University Hospital, SE-581 85 Linköping - Sweden - Phone: +4613101038566 - e-mail: Jahan.Abtahi@regionostergotland.se

Submitted: February 26, 2015 - Modification: May 22, 2015 - Accepted: May 22, 2015

\section{ABSTRACT}

\begin{abstract}
bjective: There is strong evidence of a link between the use of systemic bisphosphonates (BPs) and osteonecrosis of the jaw, especially in cancer patients. Among risk factors for BRONJ, tooth extraction and immune suppressive drugs seem to have significant role on bone healing. Therefore, the importance of these parameters in development of BRONJ was reviewed in this retrospective study in two maxillofacial surgery units. Material and Methods: From 2007 to 2012, 46 patients on bisphosphonate who had developed oral bony lesions participated in this study. The pharmacological exposure, comorbidities, maxillofacial findings, types of treatment and outcome data were collected from clinical and radiological records. Results: The most frequently used BP was alendronate (67\%). Tooth extraction was reported in $61 \%$ of patients with BRONJ. Systemic corticosteroids were prescribed in 35 cases $(76 \%)$ as an adjuvant for BP. Patients on corticosteroids had a lower probability of bony lesion healing $(p<0.05)$ than patients without corticosteroids. Of the 46 patients who underwent conservative treatments, only ten were completely healed $(21 \%)$. Conclusions: Beside tooth extraction, corticosteroids were shown to be an implant risk factor for low rate of bone healing and hence the development of BRONJ. The outcome of conservative treatment was uncertain and this emphasizes the importance of prevention.
\end{abstract}

Keywords: Bisphosphonates. Jaw. Bisphosphonate-associated osteonecrosis of the jaw. Oral health. Osteoporosis.

\section{INTRODUCTION}

Bisphosphonates are antiresorptive drugs that act specifically on osteoclasts, thereby maintaining bone density and strength ${ }^{23}$. These compounds are used in many clinical settings, including prevention and treatment of primary and secondary osteoporosis, Paget's disease of the bone, hypercalcaemia, multiple myeloma and osteolysis associated with bone metastases of malignant tumors ${ }^{10}$. Specially among patients with cancer, intravenous bisphosphonate therapy has been linked to osteonecrosis of the jaw ${ }^{5,12,18}$. This condition is defined as a non-healing wound with exposed bone, persisting for more than 8 weeks in a patient receiving bisphosphonates and with no history of local radiation therapy ${ }^{19}$. For patients on bisphosphonates, surgical procedures exposing the jaw bone to the oral cavity will increase the risk of BRONJ5,12,18. The calculated frequency of bisphosphonate-related osteonecrosis of the jaw (BRONJ) after tooth extraction in high risk patients ranges from $7 \%$ to $9 \%{ }^{19}$. However, many of these cases occur spontaneously (without any apparent treatment, trauma, or concurrent disease), and most of these have been reported with the use of bisphosphonates for a duration of 1.5 to 3 years 8 .

The pathophysiological basis of BRONJ is controversial. Previously, this condition was defined as a complication of head and neck radiotherapy ${ }^{6}$. Hence the name osteonecrosis is normally used for sterile bone death, typically due to impaired blood supply. At the time, it was speculated that bisphosphonates could cause necrotic bony lesions 
due to their effects on blood vessels of the bone, possibly by inhibition of the vascular endothelial growth factor (VEGF) ${ }^{7,25}$. More recently, it has been suggested that BRONJ does not begin as a form of classical osteonecrosis, but in fact it is osteomyelitis from the beginning ${ }^{4,11}$. Microbial contamination such as actinomyces seems to play an important role in maintaining osteomyelitic wounds ${ }^{21,26}$.

Since jaw bone containing bisphosphonates will be resorbed slowly, it is conceivable that bacterially contaminated bone cannot be removed fast enough to prevent the development of chronic osteomyelitis. This hypothesis is supported by the observation that similar lesions appear after treatment with an anti-RANKL antibody that reduces osteoclast recruitment ${ }^{29}$. Recently, it was shown in a rat model that the immediate mucoperiosteal coverage of extraction sockets prevents the development of BRONJ1.

Regarding clinical findings, four stages of bisphosphonate-induced osteonecrosis of the jaw can be identified. Early stages are characterized by exposed necrotic bone, with or without symptoms; whereas, in the later stages, the presence of pathologic fractures, extraoral fistulae and exposed necrotic bone extending beyond the region of the alveolar bone may be observed ${ }^{3}$.

The prevalence of BRONJ continues to increase worldwide owing to greater awareness of bone health ${ }^{20}$. Therefore, a clearer understanding of the pathogenesis and management strategies for BRONJ is necessary. The concurrent administration of bisphosphonate and corticosteroids, as in many patients being treated for multiple myeloma, can increase the risk of BRONJ. The importance of corticosteroids in the development of BRONJ in animal models has been discussed in several studies $^{17,27}$. The main hypothesis of the present study was that patients receiving both BPs and corticosteroids had a higher risk of developing BRONJ. In addition, the success rate and outcome of conservative approach was also reviewed.

\section{MATERIAL AND METHODS}

\section{Identification of study population}

Departments of oral and maxillofacial surgery in both Bergen (Norway) and Linköping (Sweden) provide health care to approximately half a million patients. In these regions, (approximately) $15 \%$ of the populations are treated with bisphosphonate per year because of various reasons. We used maxillofacial health databases to identify all patients diagnosed with bisphosphonate-related bony exposure of the jaw between 2007 and 2012. Because of the lack of a specific diagnosis code for bisphosphonate-related osteonecrosis of the jaw prior to 2012, we selected cases from the databases that were diagnosed by the following criteria: patient $a$, on bisphosphonate; b, with bony exposure (more than 8 weeks); c, with osteomyelitis diagnosis. This was followed by review of the medical and radiological records to confirm the diagnosis of BRONJ in the identified cases. Thus, 46 cases of BRONJ were found. Orthopanoramic photo and CT-scan showed radiolucency in connection with bony lesions in all patients. No other abnormality was found. All patients in this study had received conservative treatment, consisting of local wound care, oral antibiotics and minor surgical debridement. BRONJ was diagnosed according to the Task Force on bisphosphonate-related osteonecrosis of the jaw, as defined by the American Association of Oral and Maxillofacial Surgeons (AAOMS) ${ }^{3}$. These include: a) exposed bone in the maxillofacial region for a period of eight weeks or more, b) current or previous treatment with bisphosphonates, and c) no prior history of radiation therapy of the jaw. The staging of the disease was made according to the recommendations of the AAOMS 2014, as outlined below ${ }^{22}$.

Stage 0 - no clinical evidence of necrotic bone but nonspecific clinical findings, radiographic changes, and symptoms;

Stage 1 - exposed and necrotic bone or fistulas that probe to bone in patients who are asymptomatic and have no evidence of infection;

Stage 2 - exposed and necrotic bone or fistulas that probe to bone associated with infection as evidenced by pain and erythema in the region of exposed bone with or without purulent drainage;

Stage 3 - exposed and necrotic bone or a fistula that probes to bone in patients with pain, infection, and $\geq 1$ of the following: exposed and necrotic bone extending beyond the region of alveolar bone (i.e., inferior border and ramus in mandible, maxillary sinus, and zygoma in maxilla) resulting in pathologic fracture, extraoral fistula, oral antral or oral nasal communication, or osteolysis extending to inferior border of the mandible or sinus floor.

\section{Exclusion criteria}

Patients with primary or metastatic tumors or a history of trauma in the maxillofacial region were excluded, as these conditions often induce inflammation of the jaw. In addition, all patients with a history of radiation therapy were excluded from analysis. After the initial review, 48 cases of BRONJ were identified (stage 0-III). Two patients with stage 0 (diagnosed by radiological findings with no history of bone exposure) were excluded from the study.

\section{Confounding factors}

Data on age, gender, pharmacological exposure 
(type of bisphosphonate and duration), coadministration of corticosteroids, maxillofacial findings, types of treatment and outcomes were collected from the available clinical and radiological records. The treatment outcomes were classified into two categories: (1) complete healing with a covering of normal mucosa or (2) mucosal ulceration. All patients with ulceration had exposed bone.

\section{Statistics}

A descriptive statistical analysis was conducted and the results expressed as percentages or mean values. The main hypothesis was that patients receiving $\mathrm{BPs}$ and corticosteroids had a higher risk of developing BRONJ. This was examined through statistical testing (Fisher's exact test), and $p<0.05$ was considered significant.

\section{RESULTS}

\section{Incidence and bisphosphonate}

Data was collected from 38 females and 8 males aged between 57 and 99 years. None of the patients were confirmed as having developed ONJ due to trauma in the maxillofacial region. For all bisphosphonate users, the incidence of BRONJ per 100,000 person-years was 0.1 cases. The majority of the patients were on alendronate $(n=31)$, pamidronate $(n=3)$, ibandronate $(n=2)$ or zoledronate $(n=9)$. One patient received pamidronate and ibandronate $(n=1)$. Patients in Bergen were more likely to receive zoledronate and alendronate, while patients in Linköping received alendronate and pamidronate. The main reason for receiving BP treatment was osteoporosis for females and multiple myeloma for males. A total of 13 patients ( $28 \%$ ) were on IV bisphosphonates. The overall onset of BRONJ was earlier in the IV BP group (median, 2.5 years) compared to the oral BP group (median, 6 years). At the end of the study period, only $21 \%$ of lesions had healed.

\section{Mucosal trauma ulceration vs. spontaneous ulceration}

This condition was observed more frequently in the mandible $(n=37)$ than in the maxilla $(n=8)$. One patient had BRONJ in both the upper and lower jaw. Exposed alveolar bone occurs spontaneously (13\%) or becomes evident following tooth extraction $(61 \%)$ or dental implant $(9 \%)$. The presence of a removable oral prosthesis (full or partial) was recorded in 8 patients $(17 \%)$.

\section{Corticosteroids}

Most patients with BRONJ presented in stage II $(n=35)$ or stage I $(n=8)$. Of the overall 46 patients with BRONJ, 38 did not heal during the observation period, including 16 who died from the disease. The systemic corticosteroid prednisolone was being prescribed ( 10 to $20 \mathrm{mg}$ per day) in 35 cases (76\%). Of these, 30 patients ( $86 \%$ ) had non-healing bony lesions and the majority of them belonged to stage II. Patients on corticosteroids had a lower probability of healing $(p<0.05)$ than patients who were not on corticosteroids.

\section{DISCUSSION}

Osteonecrosis of the jaw remains a major concern for many patients using bisphosphonates. While there have been important advances in the diagnosis of BRONJ, the etiology and epidemiology of the disease are still poorly understood. Patients from two maxillofacial university clinics participated in this epidemiological study. Both departments have similar treatment strategies and provide care to almost one million patients in total.

Patients at high risk of developing BRONJ are those with malignant disease receiving high doses of intravenous bisphosphonate therapy and/or with a history of chemotherapy or concomitant use of systemic corticosteroids ${ }^{24}$. Chronic glucocorticoid therapy has adverse effects on bone metabolism, including impaired osteoblastic differentiation and function. Furthermore, the additional immunosuppressive and antiangiogenic effects of glucocorticoids can play an important role in the development of BRONJ20,22. In the current study, the systemic corticosteroid prednisolone was being prescribed for 35 patients (76\%) and, of these, 30 patients $(86 \%)$ had exposed bone at the end of the study period. However, this does not mean that a patient who has exposed bone suffers from the disease. In fact, many patients with BRONJ have no symptoms. Another risk factor for the development of ONJ was tooth extraction. In the current study, a history of tooth extraction was recorded in 28 patients with ONJ $(60 \%)$, which is in line with previous studies ${ }^{5,14,18}$.

The incidence of ONJ is estimated to be $1-12 \%$ in cancer patients receiving high-dose intravenous bisphosphonates ${ }^{5,18}$. The frequency of ONJ in bone malignancy cases, especially in those treated with intravenous bisphosphonates, was found to be 1 in $100^{19}$. If tooth extractions were performed, the calculated frequency of ONJ increased to 1 in $10^{19}$. In a retrospective study, Wang, et al. ${ }^{30}$ (2007) found that the incidence of ONJ associated with intravenous bisphosphonates was at least 3.8 per 100 patients with multiple myeloma, 2.5 per 100 patients with breast cancer, and 2.9 per 100 patients with prostate cancer. In osteoporosis patients, ONJ is rare and the incidence may not be greater than the natural background incidence of the condition. Epidemiological studies have 
indicated an estimated incidence of less than one case per 100,000 person-years of exposure to oral amino bisphosphonates ${ }^{5,18}$. Recently, in an epidemiological study of four Scandinavian countries (Denmark, Sweden, Finland and Norway) the incidence of ONJ among BP-treated patients was estimated to be between $0.7 \%$ and $1.3 \%{ }^{15}$. Almost two-thirds of the patients with ONJ were IV-users.

In the current study, the incidence of ONJ in patients on bisphosphonate was 0.1 cases per 100,000 person-years. Twenty-eight patients with BRONJ had a history of tooth extraction and $80 \%$ of the patients had a non-healing bony lesion at the end of the study period. Similar findings have been reported by others ${ }^{14,18}$.

Various hypotheses have been proposed for the pathophysiology of BRONJ, including bacterial contamination, bone remodelling suppression and angiogenesis suppression. In a prospective study by Stopeck, et al. ${ }^{28}$ (2010), 4,000 patients with metastatic bone disease were treated with zoledronate or denosumab, a rank ligand antibody that potently inhibits osteoclast differentiation and function. Osteonecrosis of the jaw was observed in both arms of the study ( $2 \%$ in patients treated with denosumab and $1.4 \%$ in patients treated with zoledronic acid). Thus, it appears that reduced resorptive activity is a key factor behind the reduced ability of these lesions to heal.

In addition to these hypothetical mechanisms, another recent hypothesis stated that bisphosphonates accumulate in bone in concentrations sufficient to be directly toxic to the oral epithelium. This would result in diminished soft-tissue healing, leading to secondary infection of the underlying bone. However, the view that BRON] starts with soft-tissue damage has prevailed. Recently, in a rat model of ONJ following tooth extraction, a high dose of alendronate $(200 \mu \mathrm{g} / \mathrm{kg})$ did not cause ONJ-like lesions ${ }^{2}$. When calculated as dose per body weight per day, the rat dose was 100 times higher than the human dose. Again, this dose did not cause mucosal ulceration in a rat model.

The present study has several limitations. Confirmation of case status depended upon findings based on information in patients' medical documents. This condition, particularly in milder forms, is often managed by dentists, who record their findings in dental, not medical, formats. Consequently, this would result in an underestimation of the number of BRONJ cases and would therefore hamper an accurate calculation of the true incidence of cases.

A clinical staging system has been developed in order to more accurately categorize patients with BRONJ22. In the early stages, surgical debridement and coverage are expected to be successful ${ }^{16}$. Segmental osteotomies are recommended only in severe cases $^{9}$. However, this controversial treatment has a high morbidity and affects the patients' quality of life $^{13}$. In the present study, the management of patients on bisphosphonate with bony exposure was conservative, and healing occurred just in about $21 \%$ of the patients. The difficulty in treating BRONJ has highlighted the importance of prevention.

\section{CONCLUSION}

Most of the BRONJ patients receiving corticosteroids had a non-healing bony lesion at the end of the present study period. In this retrospective study, the majority of patients had exposed bone despite conservative therapy. The difficulty in treating BRONJ has highlighted the importance of prevention. Further studies are still needed to identify the triggering factors associated with BRONJ and also to establish guidelines for the effective treatment of this condition.

\section{ACKNOWLEDGEMENTS}

This study was supported by the Departments of Oral and Maxillofacial Surgery at the University of Linköping \& the University of Bergen.

\section{FUNDING}

No Funding.

\section{CONFLICT OF INTEREST}

None of the authors have any financial or scientific conflicts of interest in relation to the data and conclusions presented in this manuscript.

\section{ETHICAL APPROVAL}

The study was approved by the Regional Committee for Ethics in Linköping, Sweden 2014/490-31. Approval has also been given by the authorities to review the patients' medical and radiological records.

\section{REFERENCES}

1- Abtahi J, Agholme F, Aspenberg P. Prevention of osteonecrosis of the jaw by mucoperiosteal coverage in a rat model. Int J Oral Maxillofac Surg. 2013;42:632-6.

2- Abtahi J, Agholme F, Sandberg O, Aspenberg P. Bisphosphonateinduced osteonecrosis of the jaw in a rat model arises first after the bone has become exposed. No primary necrosis in unexposed bone. J Oral Pathol Med. 2012;41:494-9.

3- Advisory Task Force on Bisphosphonate-Related Ostenonecrosis of the Jaws, American Association of Oral and Maxillofacial Surgeons. American Association of Oral and Maxillofacial Surgeons position paper on bisphosphonate-related osteonecrosis of the jaws. J Oral Maxillofac Surg. 2007;65:369-76. 
4- Aspenberg P. Osteonecrosis of the jaw: what do bisphosphonates do? Expert Opin Drug Saf. 2006;5:743-5.

5- Barasch A, Cunha-Cruz J, Curro FA, Hujoel P, Sung AH, Vena $D$, et al. Risk factors for osteonecrosis of the jaws: a case-control study from the CONDOR dental PBRN. J Dent Res. 2011;90:43944.

6- Basu N, Reid DM. Bisphosphonate-associated osteonecrosis of the jaw. Menopause Int. 2007;13:56-9.

7- Bigi MM, Escudero ND, Ubios AM, Mandalunis PM. Evaluation of vascular endothelial growth factor (VEGF) in interradicular bone marrow in olpadronate treated animals. Acta Odontol Latinoam. 2010;23:265-9.

8- Bilezikian JP. Osteonecrosis of the jaw - do bisphosphonates pose a risk? N Engl J Med. 2006;355:2278-81.

9- Carlson ER, Basile JD. The role of surgical resection in the management of bisphosphonate-related osteonecrosis of the jaws. J Oral Maxillofac Surg. 2009;67:85-95.

10- Coleman RE. Bisphosphonates: clinical experience. Oncologist. 2004;9:14-27.

11- Dodson TB, Raje NS, Caruso PA, Rosenberg AE. Case records of the Massachusetts General Hospital. Case 9-2008. A 65-yearold woman with a non-healing ulcer of the jaw. N Engl J Med. 2008;358:1283-91.

12- Fellows JL, Rindal DB, Barasch A, Gullion CM, Rush W, Pihlstrom $D J$, et al. ONJ in two dental practice-based research network regions. J Dent Res. 2011;90:433-8.

13- Filleul O, Crompot E, Saussez S. Bisphosphonate-induced osteonecrosis of the jaw: a review of 2,400 patient cases. J Cancer Res Clin Oncol. 2010;136:1117-24

14- Hoff AO, Toth B, Hu M, Hortobagyi GN, Gagel RF. Epidemiology and risk factors for osteonecrosis of the jaw in cancer patients. Ann N Y Acad Sci. 2011;1218:47-54.

15- Krüger TB, Sharikabad MN, Herlofson BB. Bisphosphonaterelated osteonecrosis of the jaw in four Nordic countries and an indication of under-reporting. Acta Odontol Scand. 2013;71:138690.

16- Lemound J, Eckardt A, Kokemüller H, von See C, Voss PJ, Tavassol F, et al. Bisphosphonate-associated osteonecrosis of the mandible: reliable soft tissue reconstruction using a local myofascial flap. Clin Oral investing. 2011;16:1143-52.

17- López-Jornet P, Camacho-Alonso F, Martínez-Canovas A Molina-Miñano F, Gómez-García F, Vicente-Ortega V. Perioperative antibiotic regimen in rats treated with pamidronate plus dexamethasone and subjected to dental extraction: a study of the changes in the jaws. J Oral Maxillofac Surg. 2011;69:2488-93.
18- Marx RE, Sawatari Y, Fortin M, Broumand V. Bisphosphonateinduced exposed bone (osteonecrosis/osteopetrosis) of the jaws: risk factors, recognition, prevention, and treatment. J Oral Maxillofac Surg. 2005;63:1567-75

19- Mavrokokki T, Cheng A, Stein B, Goss A. Nature and frequency of bisphosphonate- associated osteonecrosis of the jaws in Australia. J Oral Maxillofac Surg. 2007;65:415-23.

20- Mazziotti G, Canalis E, Giustina A. Drug-induced osteoporosis: mechanisms and clinical implications. Am J Med. 2010;123:87784.

21- Naik NH, Russo TA. Bisphosphonate-related osteonecrosis of the jaw: the role of actinomyces. Clin Infect Dis. 2009;49:1729-32. 22- Ruggiero SL, Dodson TB, Fantasia J, Goodday R, Aghaloo T, Mehrotra B, et al. American Association of Oral and Maxillofacial Surgeons position paper on medication-related osteonecrosis of the jaw - 2014 update. J Oral Maxillofac Surg. 2014;72:1938-56. 23- Russell RG, Rogers MJ. Bisphosphonates: from the laboratory to the clinic and back again. Bone. 1999;25:97-106.

24- Rutkowski JL. Combined use of glucocorticoids and bisphosphonates may increase severity of bisphosphonate-related osteonecrosis of the jaw. J Oral Implantol. 2011;505:1336-7.

25- Santini D, Vincenzi B, Avvisati G, Dicuonzo G, Battistoni $F$, Gavasci $M$, et al. Pamidronate induces modifications of circulating angiogenetic factors in cancer patients. Clin Cancer Res. 2002;8:1080-4.

26- Sedghizadeh PP, Kumar SK, Gorur A, Schaudinn C, Shuler CF, Costerton JW. Identification of microbial biofilms in osteonecrosis of the jaws secondary to bisphosphonate therapy. J Oral Maxillofac Surg. 2008;66:767-75.

27- Sonis ST, Watkins BA, Lyng GD, Lerman MA, Anderson KC. Bony changes in the jaws of rats treated with zoledronic acid and dexamethasone before dental extractions mimic bisphosphonaterelated osteonecrosis in cancer patients. Oral Oncol. 2009;2:16472.

28- Stopeck AT, Lipton A, Body JJ, Steger GG, Tonkin K, de Boer $\mathrm{RH}$, et al. Denosumab compared with zoledronic acid for the treatment of bone metastases in patients with advanced breast cancer: a randomized, double-blind study. J Clin Oncol. 2010;10:5132-9.

29- Taylor KH, Middlefell LS, Mizen KD. Osteonecrosis of the jaws induced by anti-RANK ligand therapy. $\mathrm{Br}$ J Oral Maxillofac Surg. 2010;48:221-3.

30- Wang EP, Kaban LB, Strewler GJ, Raje N, Troulis MJ. Incidence of osteonecrosis of the jaw in patients with multiple myeloma and breast or prostate cancer on intravenous bisphosphonate therapy. J Oral Maxillofac Surg. 2007;65:1328-31. 